\title{
La dieta y fauna de endoparásitos del pejesapo Gobiesox marmoratus Jenyns, 1842 (Pisces: Gobiesocidae) en el litoral central de Chile están conectadas pero no correlacionadas
}

\author{
Feeding habits and endoparasite fauna of the clingfish Gobiesox marmoratus Jenyns, 1842 \\ (Pisces: Gobiesocidae) on the central coast of Chile are intertwined, but not correlated
}

\section{CECILIA PARDO-GANDARILLAS, FÉLIX GARCÍAS \& MARIO GEORGE-NASCIMENTO}

Departamento Ecología Costera, Facultad de Ciencias, Universidad Católica de la Santísima Concepción, Casilla 297, Concepción Chile; e-mail: gandarillas@ucsc.cl

\begin{abstract}
RESUMEN
Se documenta una alta similitud en la composición de la fauna parasitaria y de la dieta de 108 ejemplares del pejesapo Gobiesox marmoratus Jenyns, 1842 recolectados de la costa de la zona centro-sur de Chile, desde tres localidades separadas por al menos $400 \mathrm{~km}$ de su(s) vecina(s). La longitud total de los pejesapos fue similar entre localidades y su parasitofauna estuvo compuesta por 16 taxa de metazoos (incluyendo cinco Myxozoa). En el 38,8 \% de los 108 pejesapos se encontraron parásitos, en tanto que en el 32,4\% de los mismos ejemplares había contenido estomacal, en los que se determinaron 37 ítems presa. La aún más baja ocurrencia conjunta de parásitos y presas imposibilitó el uso de parejas de datos parasitarios y dietarios, para evaluar, por ejemplo, si había correlación entre la amplitud dietaria y la riqueza parasitaria. Aunque la composición de la fauna de endoparásitos necesariamente depende de la composición de la dieta de los pejesapos, encontramos muy pocos parásitos en uno de los sitios de estudio. Es decir, las propiedades numéricas de este sistema hospedador-parásito limitaron la posibilidad de hallar asociaciones entre ambos tipos de variables. Tanto la composición de la dieta como de las infracomunidades de parásitos variaban con la ontogenia del huésped. La dieta de los pejesapos juveniles y adultos consistía principalmente de anfípodos, crustáceos decápodos y moluscos en las tres localidades. La similitud de los descriptores numéricos de la dieta y de las infracomunidades (abundancia total, diversidad y riqueza) entre las localidades, en parte puede deberse al escaso tamaño corporal máximo alcanzado por G. marmoratus, a la escasa longitud total del tracto digestivo, a las diferencias en las tasas de ingreso y tiempo de residencia de parásitos y presas, al nivel de resolución taxonómica alcanzado en presas y/o parásitos, y al efecto de decidir excluir o no de los análisis a las especies raras.
\end{abstract}

Palabras clave: dieta, comunidades parasitarias, Gobiesox marmoratus, ontogenia, Chile.

\begin{abstract}
A high similarity is documented in the composition of the endoparasitic fauna and the diet of the clingfish $G$. marmoratus Jenyns, 1842, between three localities off the central coast of Chile separated about $400 \mathrm{~km}$ from their nearest neighbor(s). Clingfish in the samples were similar between localities in total body length. The parasite fauna was composed by 16 metazoan taxa (including five Myxozoa). Parasites were found in $38.8 \%$ of the 108 clingfish examined, whereas $32.4 \%$ of the fish in the samples had stomach contents, where 37 prey items were recognized. The even rarer cases of co-occurrence of parasites and prey impeded the search of correlations between both type of variables, such as that between the dietary width and parasite richness. Composition of parasite infracommunities and diet changed along host ontogeny. The diet of juvenile and adult clingfish consisted mainly of amphipods, decapod crustaceans and mollusks at the three localities. The numerical descriptors of the diet and of infracommunities (total abundance, diversity and richness) are similar between the localities and along the host ontogeny. The composition of the endoparasitic fauna necessarily depends on the diet of the clingfish, but there is no statistical correlation between both variables. This can be due to the small maximum body size reached by the adults, to the short length of the digestive tract, to the differences in the rate and frequency at which parasites and prey enter and stay in the hosts, to the level of taxonomic resolution attained in prey and/or parasites, and to having decided to include or not rare prey/ parasites in the analyses.
\end{abstract}

Key words: diet, parasite communities, Gobiesox marmoratus, ontogeny, Chile. 


\section{INTRODUCCIÓN}

En los estudios en la ecología de los parásitos se denomina infracomunidad al conjunto de especies que ocupan a un individuo huésped (Holmes \& Price 1986). El progreso en el entendimiento de los procesos que originan patrones en las infracomunidades requiere una aproximación que considere la magnitud de las variaciones espaciales y temporales que las afectan (Poulin \& Valtonen 2002). Además, se requiere integrar los procesos biológicos que participan en la transmisión de los parásitos a los individuos hospedadores, como es el caso de las características del nicho trófico de los huéspedes y las de los endoparásitos transmitidos por esa vía.

En Chile se ha observado que son más frecuentes las variaciones en composición en las comunidades de parásitos de algunas especies de peces marinos, que en sus propiedades agregadas (e.g., diversidad, riqueza de especies), luego de considerar los cambios ontogenéticos de la dieta de los huéspedes sobre la abundancia de los parásitos (George-Nascimento \& Iriarte 1989, Rodríguez \& GeorgeNascimento 1996, Balboa \& George-Nascimento 1998, George-Nascimento 2000, Garcías et al. 2001, Díaz \& George-Nascimento 2002, Muñoz et al. 2001, 2002). En los pocos estudios que integran los aspectos de la dieta del hospedador y de las comunidades parasitarias, no se ha evaluado estadísticamente si hay correlaciones significativas entre descriptores de la dieta y de las comunidades parasitarias (excepto George-Nascimento 1987), tanto a lo largo de la ontogenia del hospedador como entre y dentro de localidades geográficas (Zander et al. 1999, Muñoz et al. 2001, 2002, Aldana et al. 2002). Por ejemplo, en Graus nigra y en Girella laevifrons no había una clara correspondencia entre los cambios ontogénicos de la dieta y del parasitismo (Aldana et al. 2002), y en Notothenia c. f. angustata había alta similitud geográfica en la dieta pero no en el parasitismo (Muñoz et al. 2001), y en Bovichthys chilensis se encontró una escasa y compleja relación cuantitativa entre la composición de la dieta y del parasitismo (Muñoz et al. 2002). En consecuencia, hay un espectro amplio de posibilidades de combinaciones en que un parásito puede o no estar infectando a un hospedador intermedia- rio, y ser parte o no de la dieta de un determinado depredador-hospedador, en distintos sitios de estudio.

En este estudio se describe la composición de la dieta y de la fauna parasitaria de Gobiesox marmoratus, y la magnitud de sus variaciones entre localidades geográficas y entre estados ontogenéticos del huésped para explorar la eventual asociación estadística entre la composición de la dieta y de la fauna de parásitos que se transmite tróficamente.

El pejesapo G. marmoratus forma parte del ensamble de peces del intermareal (Muñoz \& Ojeda 1998, Quijada \& Cáceres 2000), y su longitud total máxima alcanza aproximadamente $\operatorname{los} 16 \mathrm{~cm}$ al estado adulto (Chirichigno 2001). Mantiene una dieta carnívora durante toda su vida, aunque esta varía a lo largo de su ontogenia. Los juveniles consumen principalmente anfípodos, mientras que los adultos incorporan crustáceos decápodos (Muñoz \& Ojeda 1997, 1998). Su distribución geográfica abarca desde Bahía Independencia (Perú) hasta el extremo austral de Chile (Mann 1954), y no hay registros de parásitos para esta especie de pez en Chile. En este estudio se describen por primera vez en Chile las variaciones geográficas de la dieta de esta especie, y se discute cómo las características del hospedador, de la dieta y de las comunidades de parásitos, tales como su composición o diversidad, limitan la posibilidad de detectar si hay correlación entre, por ejemplo, la riqueza parasitaria y la amplitud dietaria, que se espera que podrían estar naturalmente asociadas positivamente.

\section{MATERIALES Y MÉTODOS}

Entre julio y noviembre del año 2000 se recolectaron manualmente 108 ejemplares de $G$. marmoratus desde pozas intermareales de las localidades de El Tabo $\left(\mathrm{n}=25 ; 33^{\circ} 27^{\prime} \mathrm{S}\right.$, $\left.71^{\circ} 38^{\prime} \mathrm{O}\right)$, Burca $\left(\mathrm{n}=62 ; 36^{\circ} 32^{\prime} \mathrm{S}, 72^{\circ} 55^{\prime} \mathrm{O}\right)$ y Metri ( $\left.=21 ; 41^{\circ} 37^{\prime} \mathrm{S}, 72^{\circ} 40^{\prime} \mathrm{O}\right)$. Cada uno de los ejemplares fue preservado a $-20{ }^{\circ} \mathrm{C}$, determinándose su longitud total con $1 \mathrm{~mm}$ de precisión, luego de ser descongelados.

Se practicaron disecciones completas para una observación sectorizada de los órganos y musculatura. Los ejemplares pejesapos que presentaban gónadas se clasificaron como adultos y los sin gónadas como juveniles. Se examina- 
ron el tubo digestivo y las otras vísceras, bajo microscopio estereoscópico para buscar endoparásitos e ítems presa. En forma similar se examinaron las aletas, cavidad bucal y cámara branquial para buscar ectoparásitos. La presencia de ectoparásitos fue registrada, pero los datos no fueron incorporados a los análisis de su relación con la dieta del hospedador. Cada órgano y su contenido fueron tamizados bajo agua potable a presión, en un cilindro plástico cuyo fondo consistía en una malla de $0,33 \mathrm{~mm}$ de luz y el material retenido en el tamiz fue examinado. Los parásitos y presas fueron guardados separadamente en frascos con formalina al $10 \%$, debidamente rotulados. La cuantificación y determinación taxonómica de los parásitos fueron realizadas según los criterios de Zdzitowiecki (1997) para los digeneos, Yamaguti (1961) para nematodos, Zdzitowiecki (1991) para acantocéfalos y a Lom \& Dyková (1992) para los myxozoos. El nivel de resolución taxonómica alcanzado en las presas dependió en gran parte del grado de digestión en que se encontraban. Para ello se consultó a Retamal (1981) para los crustáceos decápodos, Jaramillo (1982) para los isópodos, al Dr. Martin Thiel (comunicación personal) para anfípodos e isópodos, Valdovinos (1999) para moluscos y Chirichigno (1974) para peces.

La prevalencia (porcentaje de individuos huéspedes parasitados) y la abundancia (número de parásitos de cada taxon/número total de huéspedes examinados, ver Margolis et al. 1982) fueron calculados para cada taxon parasitario. A nivel infracomunitario, los descriptores utilizados fueron la riqueza (número de taxa parasitarios por hospedador parasitado, ver Holmes \& Price 1986), la intensidad (número de individuos parásitos de cualquier taxon por huésped parasitado), la prevalencia total (porcentaje de hospedadores parasitados por algún taxon parasitario), la abundancia total (número total de individuos parásitos en cada infracomunidad), la dominancia y la diversidad (calculadas mediante el índice de Brillouin, ver Magurran 1988). Los individuos presa fueron contados y pesados (g), para luego calcular el porcentaje del número total de las presas $(\% \mathrm{~N})$, el porcentaje del peso total en los contenidos estomacales $(\% \mathrm{P})$, y la frecuencia porcentual de ocurrencia en el total de estómagos con contenido estomacal (\%F, Hyslop 1980). Los descriptores cuantitativos de la dieta fueron la am- plitud dietaria (número de taxa en el contenido estomacal de cada pejesapo), el número de presas (en cada ejemplar), diversidad y dominancia (calculadas mediante el índice de Brillouin, ver Magurran 1988).

El análisis de la variación conjunta en la composición de la dieta y del endoparasitismo contempló primero una ordenación de los datos, y luego, análisis de la varianza de los puntajes de las ordenaciones entre localidades. En la ordenación de los parásitos se excluyó a los ectoparásitos y a los endoparásitos que aparecieron menos de tres veces en el total de huéspedes (Gonocerca sp., Aporocotyle sp., larva Digeneo, Arhythmacanthidae gen. sp., Philometridae gen. sp., Leptotheca sp., Kudoa sp.). En la ordenación de la dieta se excluyó a las presas con menos de dos ocurrencias (21 taxa). En suma, se utilizaron análisis de correspondencia con efecto de arco corregido (DCA) para la ordenación de siete taxa endoparasitarios y 37 infracomunidades (10 infracomunidades en El Tabo, 24 en Burca y tres en Metri), y para la ordenación de 16 ítems presas y 32 pejesapos con contenido alimentario (nueve de El Tabo, 11 de Burca y 12 de Metri). La composición de las infracomunidades y de los contenidos alimentarios se representa a través de los puntajes de los parásitos e ítems presa en el primer y segundo eje de las ordenaciones, cuyas unidades son desviaciones estándar (x 100) de la tasa de reemplazo de los taxa a lo largo del gradiente. Todas las ordenaciones se realizaron sobre las matrices de presencia-ausencia. El puntaje de las infracomunidades en el primer y segundo gradiente composicional de las ordenaciones fue comparado entre localidades a través de la prueba de Kruskal-Wallis, mientras que el de los contenidos estomacales fue comparado mediante análisis de la covarianza, considerando como fuente de variación la localidad y la longitud total del pez como covariable. Además, y con el objeto de contrastar los resultados obtenidos con el mayor nivel de resolución taxonómica en las presas, se realizó una prueba de Chi-cuadrado para comparar la composición de la dieta entre las localidades, pero con un bajo nivel de resolución taxonómica.

Se usaron tablas de contingencia para comparar entre localidades de muestreo, la prevalencia por cada uno y el total de los taxa parasitarios, y se evaluaron estadísticamente mediante la prueba de Chi-cuadrado. La longi- 
tud total, riqueza, abundancia total, diversidad y dominancia fueron comparadas entre localidades a través de la prueba de Kruskal-Wallis. Se calculó el coeficiente de correlación de Spearman $\left(\mathrm{r}_{\mathrm{s}}\right)$ para evaluar la asociación entre la longitud total del huésped con los descriptores infracomunitarios (Zar 1996).

\section{RESULTADOS}

La longitud total de los pejesapos en este estudio fluctuó entre 3,1 y $12,2 \mathrm{~cm}$ y no fue significativamente diferente entre las localidades (El Tabo: $6,3 \pm 1,8, \mathrm{n}=25$; Burca: $6,0 \pm 1,4, \mathrm{n}=$ 62; Metri: 6,6 $\pm 1,7, \mathrm{n}=21$; prueba de Kruskal Wallis, $\mathrm{H}=2,88, \mathrm{P}=0,23$ ). Solo el $32,4 \%$ de los 108 ejemplares tenía contenido estomacal, y en ellos se determinaron 37 ítems presa (Tabla 1). Los anfípodos fueron el ítem presa más fre- cuente en las tres localidades, además en cada localidad se destacaron ítems como los moluscos Scurria sp., S. ceciliana y Nacella sp. en El Tabo, decápodos braquiuros, Excirolana hirsuticauda y el gastrópodo Noddilittorina araucana en Burca, e isópodos, Crepidula sp. y una especie de poliplacóforo en Metri (Tabla 1). Se encontró que no había diferencias significativas entre localidades, al comparar la composición de la dieta con un bajo nivel de resolución taxonómica en las presas $\left(\chi^{2}=4,39, \mathrm{P}=\right.$ 0,35, gl = 4, Fig. 1).

La composición de la dieta se correlacionaba significativamente con la longitud total de los pejesapos en el total de los datos $\left(\mathrm{r}_{\mathrm{s}}=0,46\right.$; $\mathrm{P}=0,006 ; \mathrm{n}=32$ ), $\mathrm{y}$ en dos localidades (Tabla 2). Sin embargo, ninguno de los descriptores cuantitativos de la dieta estuvo correlacionado con la longitud total de los pejesapos en la muestra global (amplitud o taxa de presas por
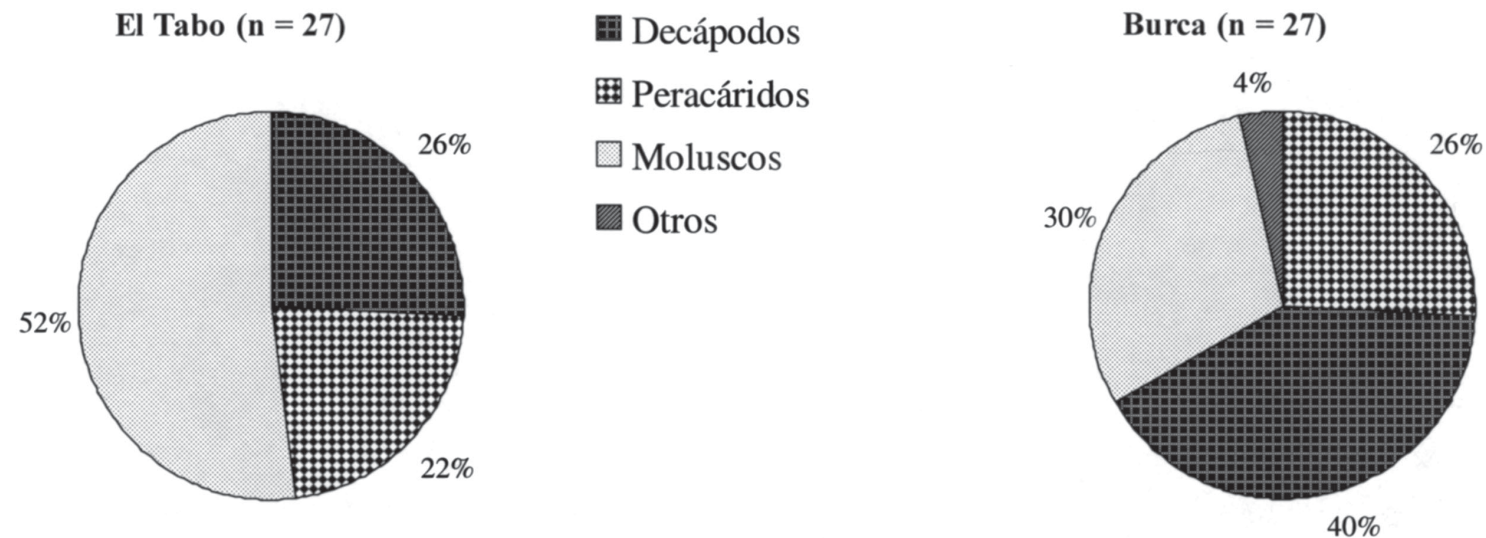

Metri $(\mathbf{n}=\mathbf{3 0})$

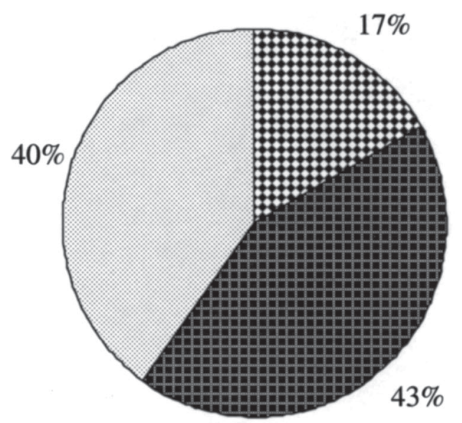

Fig. 1: Frecuencia porcentual de tipos de presas de G. marmoratus por localidades; n representa el total de las ocurrencias.

Percent frequency of prey type of G. marmoratus by localities; n represent total occurrence. 


\section{TABLA 1}

Representación porcentual de cada ítem presa de G. marmoratus con respecto al número total de presas $(\mathrm{N})$, frecuencia de ocurrencia en el total de estómagos con contenido $(\mathrm{F})$, y peso total de presas en $\mathrm{g}(\mathrm{P})$, por localidad

Percent of each prey item in the stomach contents of G. marmoratus expressed in numbers (N), occurrence frequency (F), gravimetric in $\mathrm{g}(\mathrm{P})$, according to sampling localities

\begin{tabular}{|c|c|c|c|c|c|c|c|c|c|}
\hline \multirow[t]{2}{*}{ Ítem alimentario } & \multicolumn{3}{|c|}{$\begin{array}{l}\text { El Tabo } \\
\text { (V Región) }\end{array}$} & \multicolumn{3}{|c|}{$\begin{array}{c}\text { Burca } \\
\text { (VII Región) }\end{array}$} & \multicolumn{3}{|c|}{$\begin{array}{c}\text { Metri } \\
\text { (X Región) }\end{array}$} \\
\hline & $\mathrm{N}$ & $\mathrm{F}$ & $P$ & $\mathrm{~N}$ & $\mathrm{~F}$ & $\mathrm{P}$ & $\mathrm{N}$ & $\mathrm{F}$ & $\mathrm{P}$ \\
\hline \multicolumn{10}{|l|}{ Crustacea } \\
\hline Petrolisthes sp. & & & & & & & 2,8 & 8,3 & 14,6 \\
\hline Petrolisthes violaceus & 1,2 & 8,3 & 7,6 & & & & & & \\
\hline Petrolisthes desmarestii & 1,2 & 8,3 & 2,2 & & & & & & \\
\hline Petrolisthes tuberculatus & 1,2 & 8,3 & 0,1 & & & & & & \\
\hline Allopetrolisthes spinifrons & 2,4 & 8,3 & 8,7 & & & & & & \\
\hline Allopetrolisthes angulosus & 3,6 & 8,3 & 28,3 & & & & & & \\
\hline Acanthocyclus hassleri & 1,2 & 8,3 & 0,1 & 0,9 & 9,1 & 1,1 & & & \\
\hline Acanthocyclus sp. & & & & 9,2 & 9,1 & 13,2 & 0,9 & 8,3 & 4,9 \\
\hline Hemigrapsus crenulatus & & & & & & & 0,9 & 8,3 & 2,9 \\
\hline Decapoda & 3,6 & 8,3 & 0,1 & & & & & & \\
\hline Brachyura (a) & & & & 5,5 & 27,3 & 14,3 & 0,9 & 8,3 & 0,1 \\
\hline Brachyura (b) & & & & 3,7 & 18,2 & 3,4 & 0,9 & 8,3 & 0,1 \\
\hline Amphipoda & 3,6 & 16,7 & 0,2 & 28,4 & 45,4 & 1,5 & 34,6 & 58,3 & 11,9 \\
\hline Hyale sp. & 19,1 & 25,0 & 0,2 & 22,0 & 27,3 & 0,3 & 15,0 & 16,7 & 1,1 \\
\hline Isopoda & & & & & & & 6,5 & 16,7 & 3,9 \\
\hline Isopoda (a) & & & & 0,9 & 9,1 & 0,1 & 0,9 & 8,3 & 0,1 \\
\hline Excirolana hirsuticauda & 8,3 & 8,3 & 0,1 & 16,5 & 18,2 & 22,1 & 14,0 & 8,3 & 1,0 \\
\hline \multicolumn{10}{|l|}{ Mollusca } \\
\hline Scurria sp. & 7,1 & 25,0 & 0,3 & 0,9 & 9,1 & 0,1 & & & \\
\hline Scurria scurra & 1,2 & 8,3 & 0,1 & & & & & & \\
\hline Nacella sp. & 9,5 & 16,7 & 37,2 & & & & & & \\
\hline Scurria ceciliana & 7,1 & 16,7 & 4,5 & & & & & & \\
\hline Acmea viridula & 2,4 & 8,3 & 3,3 & 0,9 & 9,1 & 0,1 & 1,9 & 8,3 & 27,2 \\
\hline Crepidula sp. & 22,6 & 8,3 & 5,4 & & & & 4,7 & 16,7 & 3,0 \\
\hline Fisurella crassa & 1,2 & 8,3 & 0,1 & & & & & & \\
\hline Fisurella maxima & 1,2 & 8,3 & 1,1 & & & & & & \\
\hline Mytilidae & & & & 0,9 & 9,1 & 0,1 & & & \\
\hline Aulacomya ater & & & & & & & 1,9 & 8,3 & 1,9 \\
\hline Nodilittorina araucana & 1,2 & 8,3 & 0,1 & 4,6 & 18,2 & 0,2 & 0,9 & 8,3 & 0,1 \\
\hline Nassarius sp. & & & & 0,9 & 9,1 & 0,1 & & & \\
\hline Tegula atra & 1,2 & 8,3 & 0,1 & & & & & & \\
\hline Chiton magnificus & & & & 0,9 & 9,1 & 0,1 & & & \\
\hline Polyplacophora (4) & & & & 0,9 & 9,1 & 2,2 & 0,9 & 8,3 & 0,1 \\
\hline Polyplacophora (1) & & & & & & & 9,3 & 25,0 & 18,5 \\
\hline Polyplacophora (2) & & & & & & & 0,9 & 8,3 & 1,9 \\
\hline Polyplacophora (5) & & & & & & & 0,9 & 8,3 & 1,0 \\
\hline Polyplacophora (3) & & & & & & & 0,9 & 8,3 & 5,8 \\
\hline \multicolumn{10}{|l|}{ Peces } \\
\hline \multicolumn{10}{|l|}{ Gobiesociformes } \\
\hline Sicyases sanguineus & & & & 2,8 & 9,1 & 40,8 & & & \\
\hline Total & 84 & 12 & 0,92 & 109 & 11 & 0,91 & 107 & 12 & 1,03 \\
\hline
\end{tabular}


pejesapo, $\mathrm{r}_{\mathrm{s}}=0,20, \mathrm{P}=0,25$; número de individuos presa por pejesapo, $\mathrm{r}_{\mathrm{s}}=0,02, \mathrm{P}=$ 0,900 ; diversidad, $\mathrm{r}_{\mathrm{s}}=0,29, \mathrm{P}=0,100$; dominancia, $\mathrm{r}_{\mathrm{s}}=-0,13, \mathrm{P}=0,460 ; \mathrm{n}=32$ en todos los casos), o en cada localidad (ver Tabla 2). Tampoco hubo variaciones significativas de ellos entre las localidades (prueba de KruskalWallis; $0,310<\mathrm{P}<0,930$ ).

Solo hubo variaciones significativas en la composición de la dieta (ontogenéticas y geográficas) en el segundo eje del DCA (primer eje, longitud total: $\mathrm{F}_{1,28}=0,05 ; \mathrm{P}=0,83$; localidad: $\mathrm{F}_{2,28}=1,56 ; \mathrm{P}=0,23$; segundo eje, longitud total: $\mathrm{F}_{1,28}=7,14, \mathrm{P}=0,01$; localidad: $\mathrm{F}_{2,28}=5,03 ; \mathrm{P}=0,01 ;$ Fig. 2A). La ordenación de presas y consumidores mostró valores propios $\lambda_{1}=0,88, \lambda_{2}=0,65$ en el primer y segundo gradiente composicional, respectivamente (Fig. 3). Estas diferencias en composición se asocian a la mayor frecuencia de moluscos en los ejemplares de menor longitud total, y a la mayor frecuencia de isópodos y anfípodos en los de mayor longitud total (Fig. 2A), entre los taxa que fueron considerados en la ordenación.

El 38,9\% de los 108 pejesapos examinados albergaba parásitos, y en ellos se recolectaron 193 individuos pertenecientes a 16 taxa: dos ectoparásitos, cuatro endoparásitos en estados larval, y cinco endoparásitos en estado adulto, además de Myxozoa pertenecientes a cinco géneros (Tabla 3).

Ninguno de los descriptores cuantitativos de las infracomunidades estuvo correlacionado con la longitud total de los pejesapos en el conjunto de las localidades (abundancia total, $\mathrm{r}_{\mathrm{s}}=0,26, \mathrm{P}=0,08$; riqueza, $\mathrm{r}_{\mathrm{s}}=0,26, \mathrm{P}=0,09$; diversidad, $\mathrm{r}_{\mathrm{s}}=0,26, \mathrm{P}=0,09$; dominancia, $\mathrm{r}_{\mathrm{s}}$ $=-0,22, \mathrm{P}=0,15 ; \mathrm{n}=42$ en todos los casos). En cambio, en cada una de las localidades, al menos uno de los descriptores cuantitativos de las infracomunidades se correlacionaba significativamente con la longitud total de los pejesapos (Tabla 2).

TABLA 2

Descriptores cuantitativos de la dieta y de las infracomunidades parasitarias de 108 ejemplares del pejesapo G. marmoratus según localidad de muestreo en la costa centro-sur de Chile; (n) número de individuos, $(\mathrm{x})$ promedio, (DE) desviación estándar, $\left(\mathrm{r}_{\mathrm{s}}\right.$ ) coeficiente de correlación de Spearman entre el descriptor y la longitud total del pejesapo (*) $\mathrm{P} \leq 0,05$

Quantitative descriptors of the diet and of parasite infracommunities of the clingfish G. marmoratus at three localities in the central-southern coast of Chile; (n) number of individuals, $(x)$ average, $(S D)$ standard deviation, $\left(r_{s}\right)$ Spearman's correlation coefficient between each descriptor and total body length of the clingfishes $(*) \mathrm{P} \leq 0,05$

\begin{tabular}{|c|c|c|c|c|c|c|c|c|c|}
\hline \multirow[t]{2}{*}{ Descriptor } & & \multicolumn{4}{|c|}{ Dieta } & \multicolumn{4}{|c|}{ Parásitos } \\
\hline & & $\mathrm{n}$ & $\mathrm{x}$ & $\mathrm{DE}$ & $\mathrm{r}_{\mathrm{s}}$ & $\mathrm{n}$ & $\mathrm{x}$ & $\mathrm{DE}$ & $\mathrm{r}_{\mathrm{s}}$ \\
\hline \multirow[t]{3}{*}{ Riqueza } & El Tabo & 9 & 2,5 & 1,2 & 0,29 & 10 & 1,3 & 0,5 & $-0,18$ \\
\hline & Burca & 11 & 2,4 & 1,4 & 0,17 & 28 & 1,6 & 1,2 & 0,27 \\
\hline & Metri & 12 & 2,4 & 1,1 & 0,20 & 4 & 1,7 & 0,9 & $0,94 *$ \\
\hline \multirow[t]{3}{*}{ Abundancia } & El Tabo & 9 & 8,3 & 5,8 & 0,13 & 10 & 13,3 & 26,9 & $0,79 *$ \\
\hline & Burca & 11 & 10,0 & 9,2 & 0,02 & 28 & 2,9 & 4,4 & $<0,01$ \\
\hline & Metri & 12 & 8,9 & 7,7 & $-0,01$ & 4 & 1,7 & 0,9 & $0,94 *$ \\
\hline \multirow[t]{3}{*}{ Diversidad } & El Tabo & 9 & 0,7 & 0,4 & 0,43 & 10 & 0,1 & 0,2 & 0,18 \\
\hline & Burca & 11 & 0,5 & 0,6 & 0,22 & 28 & 0,2 & 0,4 & 0,27 \\
\hline & Metri & 12 & 0,6 & 0,4 & 0,30 & 4 & 0,3 & 0,4 & $0,94 *$ \\
\hline \multirow[t]{3}{*}{ Dominancia } & El Tabo & 9 & 0,2 & 0,3 & $-0,54$ & 10 & 0,7 & 0,4 & 0,18 \\
\hline & Burca & 11 & 0,5 & 0,4 & $-0,14$ & 28 & 0,7 & 0,4 & $-0,22$ \\
\hline & Metri & 12 & 0,3 & 0,3 & $-0,02$ & 4 & 0,5 & 0,5 & $-0,89$ \\
\hline \multirow[t]{3}{*}{ Composición } & El Tabo & 9 & 234 & 113,2 & $0,70 *$ & 10 & 329 & 159,1 & $-0,26$ \\
\hline & Burca & 11 & 139 & 97,6 & 0,16 & 24 & 341 & 150,5 & $-0,39$ \\
\hline & Metri & 12 & 90 & 84,5 & $0,82 *$ & 3 & 225 & 155,5 & 1,0 * \\
\hline
\end{tabular}


(A)
$\diamond$ El Tabo
$\square$ Burca
$\triangle$ Metri

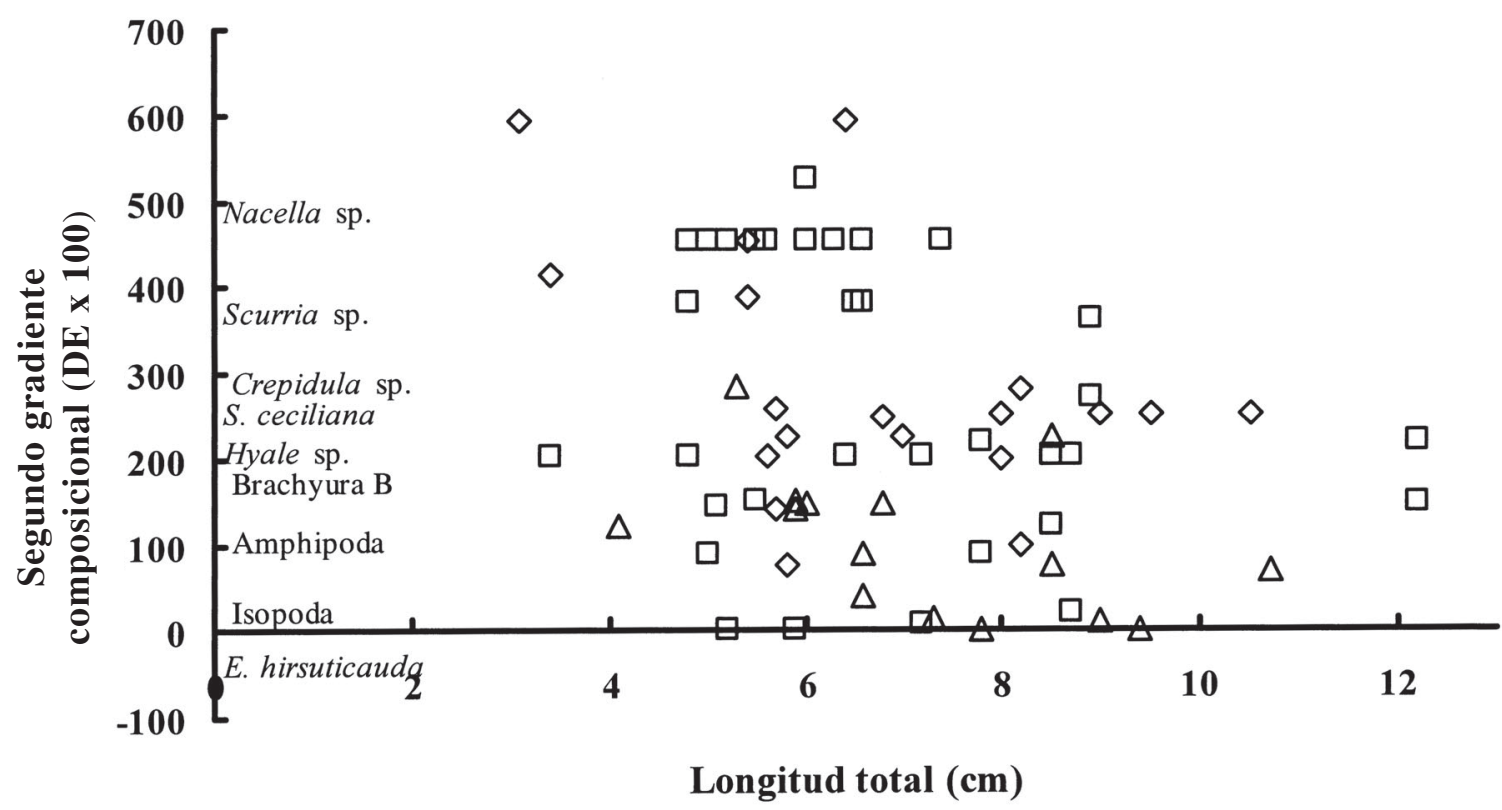

(B)

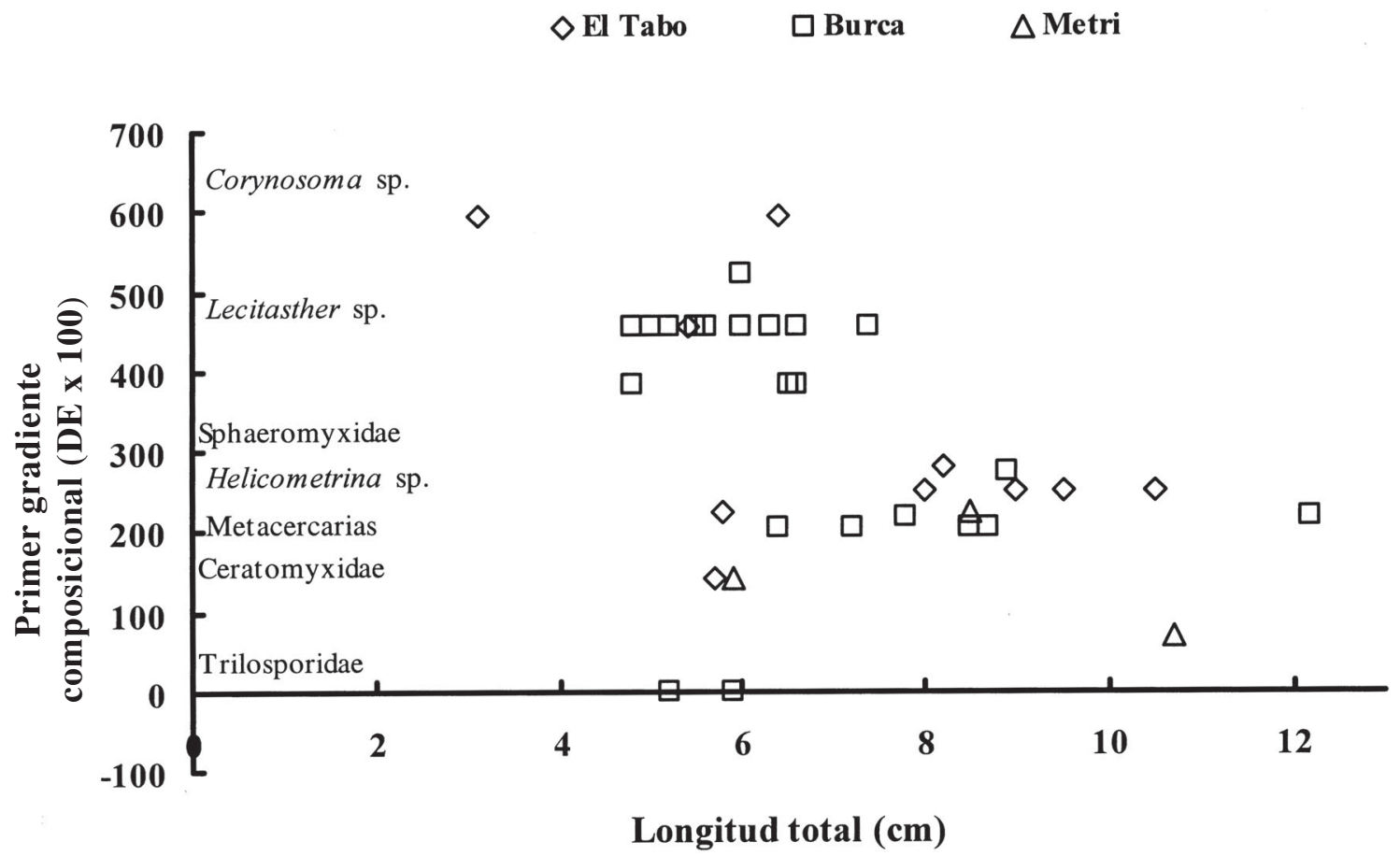

Fig. 2: (A) Relación entre la longitud total de G. marmoratus en tres localidades y el segundo gradiente composicional de la dieta; (B) relación entre el tamaño corporal de G. marmoratus en tres localidades y el primer gradiente composicional de la parasitofauna.

(A) Relationship between the total body length of G. marmoratus from three localities and athe second compositional gradient of the diet; (B) the first compositional gradient of the parasitic fauna. 
La correlación significativa de los puntajes del primer gradiente composicional del DCA con la longitud total de los hospedadores $\left(\mathrm{r}_{\mathrm{S}}=\right.$ $-0,38, \mathrm{P}=0,02, \mathrm{n}=37$; Fig. $2 \mathrm{~B}$ ) sugiere $\operatorname{los}$ cambios de la composición de las infracomunidades a lo largo de la ontogenia del huésped. La ordenación de infracomunidades y huéspedes mostró valores propios $\lambda_{1}=0,88$ y $\lambda_{2}=0,41$, en el primer y segundo gradiente de composición, respectivamente. No hubo diferencias significativas entre localidades en ninguno de los descriptores de las infracomunidades (prevalencia total: $40 \%$ en El Tabo, 35,4 \% en Burca, 19,4\% en Metri, $\chi^{2}=2,56 ; \mathrm{P}=0,28$; riqueza: $\chi^{2}=1,84$, $\mathrm{P}=0,65$; intensidad: $\chi^{2}=2,11, \mathrm{P}=0,34$, diversidad: $\chi^{2}=0,89 ; \mathrm{P}=0,64 ;$ dominancia: $\chi^{2}=$ 1,53, $\mathrm{P}=0,46$; composición: $\chi^{2}=1,57, \mathrm{P}=$ 0,$45 ; \mathrm{gl}=2$ en todos los casos; Tabla 2).

\section{DISCUSIÓN}

En resumen, no encontramos cambios significativos entre las localidades estudiadas en los descriptores numéricos de la dieta y de las infracomunidades de parásitos de G. marmoratus, pero sí en la composición de ambas, la dieta y el parasitismo, a lo largo de la ontogenia. Desafortunadamente para nuestras intenciones de análisis, los tractos digestivos en los que había parásitos no necesariamente eran en los que se encontraban presas. De hecho, en una de las localidades (Metri), casi no había macroparásitos, y solo la presencia de Myxozoa los hizo similares a los pejesapos de otras localidades.

En estudios sobre la ecología de parásitos es común que se examinen variables ecológicas del hospedador que pueden ser relevantes para entender su transmisión, distribución, abundancia y composición de sus infracomunidades (Williams et al. 1992). Los resultados de este estudio indican que los pejesapos poseen una dieta similar en las tres localidades estudiadas (Tabla 1), y refuerzan los hallazgos de Muñoz $\&$ Ojeda $(1997,1998)$ en cuanto a la incorporación de crustáceos decápodos en la dieta de los ejemplares de mayor longitud total.

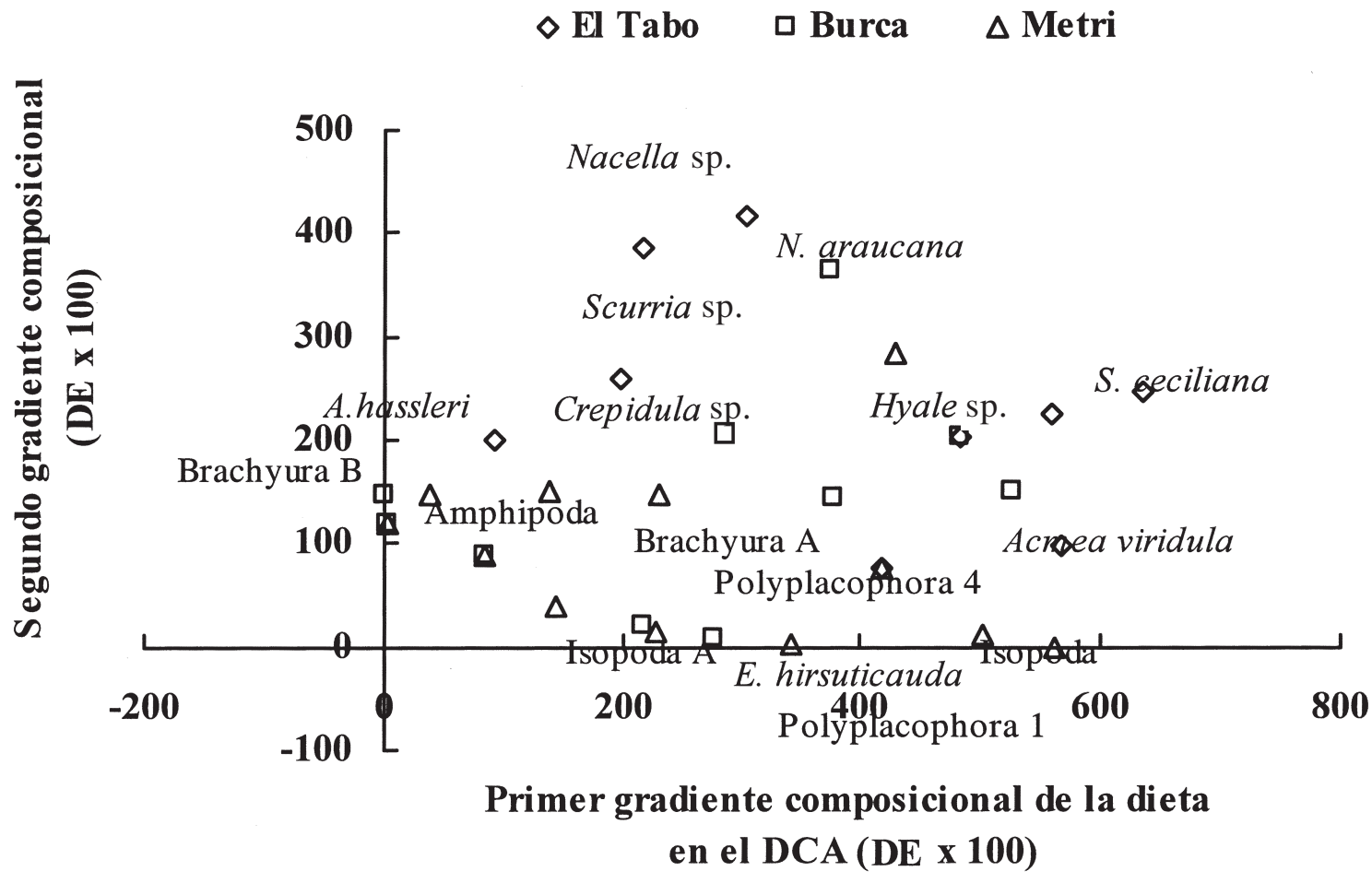

Fig. 3: Relación entre los valores del primer y segundo eje de los gradientes en composición de la dieta de G. marmoratus, según localidades geográficas.

Relationship between scores for the first and second axes of the dietary compositional gradients for G. marmoratus according to geographical localities. 
La alta semejanza entre las localidades en los descriptores cuantitativos y de composición de la dieta y de las infracomunidades parasitarias sugiere una correspondencia que vincula a los endoparásitos con las presas que comen los hospedadores, al actuar como hospedadores intermediarios o paraténicos en la transmisión (Rohde 1984, Moore 1987, Valtonen \& Julkunen 1995). Sin embargo, cuando la presencia conjunta de presas y parásitos es escasa, como lo ocurrido en la especie de hospedador de este estudio, los datos con los que se realiza el análisis de la dieta no pueden ser los mismos con los que se analizan las infracomunidades. Se deduce de estas observaciones que para abordar la pregunta sobre la correlación de la dieta y del endoparasitismo, se debiera considerar un mayor número de localidades para hospedadores con características de dieta y/o parasitismo similares a este caso. Solo en caso de seleccionar una especie de huésped cuya dieta posea características tales como una alta frecuencia de hallazgo de restos de presas identificables y parásitos, sería posible tener más parejas de datos de dieta y parasitismo, sin que se requiera un mayor número de localidades.

TABLA 3

Número de hospedadores examinados $(\mathrm{N})$, número de parásitos de cada taxon $\left(\mathrm{N}^{\circ}\right)$, prevalencia (P, \%) y abundancia (A) de 16 taxa parasitarios encontrados en 108 ejemplares de G. marmoratus recolectados desde tres localidades en la costa centro-sur de Chile

Number of hosts examined $(\mathrm{N})$, number of parasites per taxon $\left(\mathrm{N}^{\circ}\right)$, prevalence $(\mathrm{P}, \%)$ and abundance $(\mathrm{A})$ of 16 parasite taxa found in $108 \mathrm{G}$. marmoratus sampled at three localities off the central-south coast of Chile

\begin{tabular}{|c|c|c|c|c|c|c|c|c|c|}
\hline \multirow[t]{2}{*}{ Taxa } & \multicolumn{3}{|c|}{$\begin{array}{c}\text { El Tabo } \\
\text { V } \\
(\mathrm{N}=25)\end{array}$} & \multicolumn{3}{|c|}{$\begin{array}{c}\text { Burca } \\
\text { VIII } \\
(\mathrm{N}=62)\end{array}$} & \multicolumn{3}{|c|}{$\begin{array}{c}\text { Metri } \\
\mathrm{X} \\
\mathrm{N}=21)\end{array}$} \\
\hline & $\mathrm{N}^{\circ}$ & $\mathrm{P}$ & A & $\mathrm{N}^{\circ}$ & $\mathrm{P}$ & A & $\mathrm{N}^{\circ}$ & $\mathrm{P}$ & $\mathrm{A}$ \\
\hline \multicolumn{10}{|l|}{ Endoparásitos } \\
\hline Lecithaster sp. $(*)(* *)$ & 2 & 4 & 0,1 & 30 & 26 & 0,4 & & & \\
\hline Helicometrina sp. $(*)(* *)$ & 122 & 20 & 4,8 & 1 & 2 & $<0,1$ & & & \\
\hline Metacercarias (1) & & 11 & & & & -0. & & & \\
\hline Gonocerca sp. & & & & 3 & 2 & $<0,1$ & & & \\
\hline $\begin{array}{l}\text { Aporocotyle sp. } \\
\text { Digeneo (1) }\end{array}$ & & & & 2 & 3 & $<0,1$ & 1 & 5 & $<0,1$ \\
\hline \multicolumn{10}{|l|}{ Phylum Acanthocephala } \\
\hline Corynosoma sp. (1) & 2 & 8 & 0,1 & 1 & 2 & $<0,1$ & & & \\
\hline $\begin{array}{l}\text { Arhythmacanthidae gen. sp. } \\
\text { Phylum Nematoda }\end{array}$ & & & & 1 & 2 & $<0,1$ & & & \\
\hline Philometridae gen. sp. (1) & & & & 24 & 5 & 0,4 & & & \\
\hline Phylum Myxozoa & & & & & & & & & \\
\hline Sphaeromyxidae gen. sp. & & 8 & & & 5 & & & 6 & \\
\hline Ceratomyxa sp. & & 8 & & & & & & 18 & \\
\hline Leptoteca sp. & & & & & 3 & & & & \\
\hline Kudoa sp. & & & & & & & & 6 & \\
\hline Trilospora sp. & & & & & 3 & & & 6 & \\
\hline $\begin{array}{l}\text { Ectoparásitos } \\
\text { Phylum Arthropoda }\end{array}$ & & & & & & & & & \\
\hline $\begin{array}{l}\text { Pennellidae gen. sp. } \\
\text { Phylum Annelida }\end{array}$ & & & & 1 & 2 & $<0,1$ & & & \\
\hline Piscicolidae gen. sp. & 1 & 4 & $<0,1$ & & & & & & \\
\hline Total & 129 & 52 & & 63 & 66 & & 1 & 41 & \\
\hline
\end{tabular}

$(1)=$ estado larval, $(*)=$ diferencia significativa en prevalencia, $(* *)=$ diferencia significativa en abundancia. 
Todos los descriptores cuantitativos de las infracomunidades eran similares entre pejesapos juveniles y adultos. Sin embargo, la composición de las infracomunidades de endoparásitos de los pejesapos juveniles era distinta a la de los adultos, entre y dentro de cada localidad (para el caso de Burca), y en parte mantiene una correspondencia con los resultados observados en las variaciones ontogenéticas de la dieta (Fig. 2A y 2B). Estas diferencias en composición se asocian a la mayor abundancia relativa de Lecithaster sp. entre los peces más pequeños en Burca, y a la de Helicometrina sp. entre los más grandes, la mayoría ejemplares de El Tabo (Fig. 2B).

La exigua longitud máxima alcanzada por los adultos de esta especie $(12,2 \mathrm{~cm})$, limitan los valores cuantitativos que alcanzan las infracomunidades. Por otra parte, G. marmoratus es una especie que se caracteriza por tener un estómago no muy grande y un intestino muy corto, al igual que otros de sus confamiliares (Cancino \& Castilla 1988), de lo cual se presume que, pese a que la ingestión alimentaria y la transmisión de los parásitos sean procesos biológicos directamente relacionados, es poco probable que se descubra una correlación entre ambas variables, sencillamente porque la tasa de ingreso de los parásitos es menor que la de las presas, y también a que el tiempo de residencia de las presas en el tracto digestivo es menor que el de los parásitos. Además, el nivel de resolución taxonómica que se haga sobre presas también determina la posibilidad de detectar diferencias entre localidades (Fig. 1, Tabla 1). Finalmente, la baja prevalencia total y abundancia de parásitos y presas conspiran contra la posibilidad de hallar correlaciones entre estos dos procesos biológicos.

\section{AGRADECIMIENTOS}

Agradecemos al Dr. Martin Thiel (Universidad Católica de Norte, Coquimbo, Chile) por su ayuda en la determinación taxonómica de anfípodos e isópodos, y el financiamiento del Proyecto FONDECYT 1980442 y FONDAP, Programa 3, Ecología y Conservación.

\section{LITERATURA CITADA}

ALDANA M, JM PULGAR, F OGALDE \& FP OJEDA (2002) Morphometric and parasitological evidence for ontogenetic and geographical shifts in trophic status of intertidal fishes. Bulletin of Marine Science 70: $55-74$

BALBOA L \& M GEORGE-NASCIMENTO (1998) Variaciones ontogenéticas y entre años en las infracomunidades de parásitos metazoos de dos especies de peces marinos en Chile. Revista Chilena de Historia Natural 71-27-37.

CANCINO JM \& JC CASTILLA (1988) Emersion behavior and foraging ecology of the common Chilean clingfish Sicyases sanguineus (Pisces: Gobiesocidae). Journal of Natural History 22: 249. 261.

CHIRICHIGNO FN (1974) Clave para identificar los peces marinos del Perú. Instituto del Mar del Perú, Informe 44. Mar y Ciencia Imarpe, Callao, Perú. 387 pp.

CHIRICHIGNO FN (2001) Catálogo comentado de los peces marinos del Perú. Instituto del Mar del Perú, Publicación Especial, Callao, Perú. 314 pp.

DÍAZ F \& M GEORGE-NASCIMENTO (2002) Estabilidad temporal de las infracomunidades de parásitos en la borrachilla Scartichthys viridis (Valenciennes, 1836) (Pisces: Blenniidae) en la costa central de Chile. Revista Chilena de Historia Natural 75: 641649.

GARCÍAS F, R MENDOZA \& M GEORGENASCIMENTO (2001) Variación entre años de las infracomunidades de parásitos metazoos de la corvina Cilus gilberti (Pisces: Sciaenidae) en Chile. Revista Chilena de Historia Natural 74: 833-840.

GEORGE-NASCIMENTO M (1987) Ecological helminthology of wildlife animal hosts from South las America: a literature review and a search for patterns in marine food webs. Revista Chilena de Historia Natural 60: 181-202.

GEORGE-NASCIMENTO M (2000) Geographical variations in the jack mackerel, Trachurus symmetricus murphyi populations in the southeastern Pacific ocean as evidenced from the associated parasite communities. Journal of Parasitology 86: 929-932.

GEORGE-NASCIMENTO M \& JL IRIARTE (1989) Las infracomunidades de parásitos metazoos del chancharro Helicolenus lengerichi Norman, 1937 (Pisces, Scorpaenidae): un ensamble no interactivo de especies. Revista Chilena de Historia Natural 62: 217-227.

HOLMES JC \& PW PRICE (1986) Communities of parasites. En: Anderson DJ \& J Kikkawa (eds) Community ecology: patterns and process: 187-213. Blackwell Scientific Publications, Oxford, United Kingdom.

HYSLOP EJ (1980) Stomach content analysis - a review of methods and their application. Journal of Fish Biology 17: 411-429.

JARAMILLO E (1982) Taxonomy natural history and zoogeography of sand beach isopods from the coast of southern Chile. Studies on Neotropical Fauna and Environment 17: 175-194.

LOM J \& I DYKOVÁ (1992) Protozoan parasites of fishes. Elsevier Science Publishers B.V., Amsterdam, The Netherlands. 315 pp.

MAGURRAN AE (1988) Ecological diversity and its measurement. Princeton University Press, Princeton, New Jersey, USA. 175 pp.

MANN GF (1954) Vida de los peces en aguas chilenas (claves para los peces chilenos). Ministerio de Agricultura, Santiago, Chile. 342 pp.

MARGOLIS L, GW ESCH, JC HOLMES, AM KURIS \& GA SCHAD (1982) The use of ecological terms in parasitology (Report of an ad hoc committee of the 
American Society of Parasitologists). Journal of Parasitology 68: 131-133.

MOORE J (1987) Some roles of parasitic helminths in trophic interactions. A view from North America. Revista Chilena de Historia Natural 60: 159-179.

MUÑOZ AA \& FP OJEDA (1997) Feeding guild structure of a rocky intertidal fish assemblage in central Chile. Environmental Biology of Fishes 49: 471-479.

MUÑOZ AA \& FP OJEDA (1998) Guild structure of carnivorous intertidal fishes of the Chilean coast: implications of ontogenetic dietary shifts. Oecologia 114: 563-573.

MUÑOZ G, F GARCÍAS, V VALDEBENITO \& M GEORGE-NASCIMENTO (2001) Parasitofauna y alimentación de Notothenia c.f. angustata Hutton 1875 (Pisces: Nototheniidae) del intermareal de dos localidades del Golfo de Arauco. Chile. Boletín Chileno de Parasitología 56: 29-33.

MUÑOZ G, V VALDEBENITO \& M GEORGENASCIMENTO (2002) La dieta y la fauna de parásitos metazoos del torito Bovichthys chilensis Regan 1914 (Pisces: Bovichthydae) en la costa de Chile centro-sur: variaciones geográficas y ontogenéticas. Revista Chilena de Historia Natural 75: 661-671.

POULIN R \& E T VALTONEN (2002) The predictability of helminth community structure in space: a comparison of fish populations from adjacent lakes. International Journal for Parasitology 32: 1235-1243.

QUIJADA P \& C CÁCERES (2000) Patrones de abundancia, composición trófica y distribución espacial del ensamble de peces intermareales de la zona centrosur de Chile. Revista Chilena de Historia Natural 73: 739-747.

RETAMAL MA (1981) Catálogo ilustrado de los crustáceos decápodos de Chile. Gayana Zoología (Chile) 44: 1-110.
RODRÍGUEZ L \& M GEORGE-NASCIMENTO (1996) La fauna de endoparásitos metazoos del bacalao de profundidad Dissostichus eleginoides Smitt 1898 (Pisces: Nototheniidae) en Chile central: aspectos taxonómicos ecológicos y zoogeográficos. Revista Chilena de Historia Natural 69: 21-33.

ROHDE K (1984) Ecology of marine parasites. Helgoländer Meeresuntersuchungen 37: 5-33.

VALDOVINOS C (1999) Biodiversidad de moluscos chilenos: base de datos taxonómica y distribucional. Gayana Zoología (Chile) 63: 111-164.

VALTONEN ET \& M JULKUNEN (1995) Influence of the transmission of parasites from prey fishes on the composition of the parasite community of a predatory fish. Canadian Journal of Fisheries and Aquatic Sciences 52: 233-245.

WILLIAMS H, K MACKENZIE \& A MCCARTHY (1992) Parasites as biological indicators of the population biology, migrations, diet and phylogenetics of fish. Reviews in Fish Biology and Fisheries 2: 144-176.

YAMAGUTI S (1961) Systema Helminthum: the Nematodes of vertebrates. Volume X. Interscience Publishers, New York, New York, USA. 679 pp.

ZANDER CD, LW REIMER \& K BARZ (1999) Parasite communities of the Salzhaff (Northwest Mecklenburg, Baltic Sea). I. Structure and dynamics of communities of littoral fish, especially small-sized fish. Parasitology Research 85: 356-372.

ZAR JH (1996) Biostatistical analysis. Second edition. Prentice-Hall Inc., Englewood Cliffs, New Jersey, USA. 622 pp.

ZDZITOWIECKI K (1991) Antarctic Acanthocephala. Synopses of the Antarctic Benthos. Koeltz Scientific Books, Konigstein, Germany. 116 pp.

ZDZITOWIECKI K (1997) Antarctic Digenea. Synopses of the Antarctic Benthos. Koeltz Scientific Books, Konigstein, Germany. 156 pp. 